\title{
Safety and Cost of Outpatient Radiofrequency Ablation of the Slow Pathway in Patients with Atrioventricular Nodal Reentrant Tachycardia
}

\author{
K. Ching Man, DO, Steven J. Kalbfleisch, MD, John D. Hummel, MD, Brian D. Williamson, MD, \\ Vicken R. Vorperian, MD, S. Adam Strickberger, MD, Jonathan J. Langberg, MD, and Fred Morady, MD
}

$\mathbf{R}$ adiofrequency ablation of atrioventricular (AV) nodal reentrant tachycardia has been shown to be an effective and safe treatment and to have a significant cost advantage over other forms of therapy. ${ }^{1}$ In studies reported to date, patients were hospitalized for 2 to 10 days after slow pathway ablation to monitor for possible complications or a recurrence of the tachycardia., 2,3 A previous study reported that radiofrequency ablation of accessory pathways can be performed safely on an outpatient basis, ${ }^{4}$ but no prior studies evaluated the safety of outpatient radiofrequency ablation of the slow pathway in patients with AV nodal reentrant tachycardia. Therefore, the purpose of this study was to evaluate the safety and cost of performing radiofrequency catheter ablation of the slow AV nodal pathway on an outpatient basis.

The study population comprised 139 consecutive patients $(38$ men and 101 women, mean age $45 \pm 16$ years) who underwent radiofrequency ablation of the slow pathway for treatment of $A V$ nodal reentrant tachycardia at the University of Michigan Medical Center between August 30, 1991 and December 2, 1992. One hundred thirty-one patients did not have structural heart disease; 4 had coronary artery disease, and 1 each had mitral valve prolapse, mitral regurgitation or a dilated cardiomyopathy.

The procedure was performed with patients in the fasting state after discontinuation of all antiarrhythmic medications for $\geq 24$ hours and after informed written consent was obtained. Three 7 or $8 F r$ sheaths were positioned in the right femoral vein, and 3 quadripolar electrode catheters were positioned in the high right atrium, His bundle position and right ventricle. All patients received a 3,000 IU bolus of heparin intravenously after initial placement of catheters. Intravenous midazolam was used for sedation. An initial diagnostic electrophysiologic test was performed to determine the tachycardia mechanism, and the anterograde and retrograde conduction properties and refractory periods of the fast and slow AV nodal pathways.

The ablation catheter was a 7Fr bipolar or quadripolar electrode catheter with a $4 \mathrm{~mm}$ distal electrode and 2 to 5 mon interelectrode spacing (EP Technologies, Inc., Mountain View, California, or Mansfield-Scientific, Boston). Radiofrequency energy was delivered as an unmodulated sine wave at $500 \mathrm{KHz}$ (EP Technologies) between the distal electrode of the ablation catheter and a large skin electrode on the back, and was applied at power outputs of 30 to $35 \mathrm{~W}$ for 30 seconds. The application was terminated immediately in the event of coagulum formation, AV block or the occurrence of ven-

From the Department of Internal Medicine, Division of Cardiology, University of Michigan Medical Center, 1500 E. Medical Drive, B1F245, Ann Arbor, Michigan 48109. Manuscript received March 1, 1993; revised manuscript received and accepted May 28, 1993. triculoatrial block during junctional ectopy. Target sites were selected by mapping along the posteromedial tricuspid annulus near the coronary sinus ostium and searching for a multicomponent atrial electrogram or evidence of a possible slow pathway potential and an atrial:ventricular electrogram ratio $<0.5$. After successful ablation, atrial and ventricular stimulation was repeated before and during the infusion of isoproterenol at a rate of 2 to $4 \mu \mathrm{g} / \mathrm{min}$. Patients were observed in the electrophysiology laboratory for 30 minutes to rule out an acute recurrence of $A V$ nodal reentrant tachycardia.

Catheters and sheaths were removed at completion of the procedure, and manual compression was applied until complete hemostasis was achieved. If the observation period could be completed by 7 P.M., patients were observed for 3 hours in an outpatient recovery unit; otherwise, they were admitted to the hospital overnight.

Patients were scheduled for a follow-up visit 2 to 4 weeks after the procedure and were instructed to contact an investigator or their referring physician if tachycardia symptoms recurred. A follow-up electrophysiologic test 3 months after the ablation procedure was recommended in patients who had had infrequent episodes of paroxysmal supraventricular tachycardia before the ablation procedure.

Hospital billing records were reviewed to determine the hospital and professional charges in a subgroup of 40 consecutive patients. Hospital charges included room and board, electrophysiology laboratory, recovery room and laboratory tests. Professional charges included physician fees for the catheter ablation procedure, and those related to patient care.

of 139 patients who underwent radiofrequency ablation of the slow AV nodal pathway, 120 qualified for early discharge on either the day of the procedure $(n=90)$ or the next morning $(n=30)$, with successful ablation in 119 of 120 .

The reasons for continued hospitalization in 19 patients were the occurrence of a complication in 2 , ablation of the fast $A V$ nodal pathway in 6, a clinical indication for hospitalization unrelated to $A V$ nodal reentrant tachycardia in 4 , and inpatient status at the time of the ablation procedure in 7 . The 2 complications consisted of complete $A V$ block that resolved within 24 hours in 1 patient and persisted in the other, necessitating implantation of a permanent pacemaker.

No complications related to the procedure were $o b$ served or reported when patients were observed 2 to 4 weeks later. One hundred nineteen patients had no recurrence of symptomatic tachycardia during $7 \pm 3$ months of follow-up. One patient had a recurrence of tachycardia 3 weeks after the procedure, necessitating repeat slow pathway ablation. Five of 82 patients who underwent a followup electrophysiologic test 3 months after the ablation procedure were found to have inducible $A V$ nodal reentrant 
tachycardia; repeat radiofrequency ablation of the slow pathway was successful in each of these patients. No patient was found to have AV block or prolongation of the AH interval at the time of the follow-up electrophysiology test.

In 40 consecutive patients selected for the cost analysis, there were 27 outpatients and 13 admitted for an overnight hospital stay. The mean total charge for all $40 \mathrm{pa}$ tients was $\$ 10,547 \pm 1,062$, consisting of $\$ 6,267 \pm 481$ in professional fees, and $\$ 4,327 \pm 867$ in hospital charges. The mean total charge for patients treated as outpatients was $\$ 10,192 \pm 846$, consisting of $\$ 6,164 \pm 463$ in professional fees, and $\$ 4,028 \pm 710$ in hospital charges. The mean total charge for patients who had an overnight admission was $\$ 11,240 \pm 763$, consisting of $\$ 6,407 \pm 485$ in professional fees, and $\$ 4,833 \pm 946$ in hospital charges.

This study shows that it is safe to perform radiofrequency ablation of the slow pathway in low-risk patients with AV nodal re-entrant tachycardia on an outpatient basis and that this may help in reducing the total cost of the procedure. Eighty-six percent of patients with AV nodal reentrant tachycardia who underwent radiofrequency ablation of the slow pathway were thought to be appropriate candidates for early discharge and had no evidence of a complication during or within 3 hours after the procedure. No complication became apparent after discharge of these patients. The absence of any delayed complications indicates that it is safe to discharge the patient if no complications are apparent by 3 hours after the procedure.

The major concerns related to outpatient radiofrequency ablation procedures include possible complications of vascular access, AV block, pneumothorax and cardiac perforation. Because slow pathway ablation does not need arterial cannulation, the vascular complication rate would be expected to be low. Accordingly, in this study, there were no vascular complications related to vascular access.

Because delayed AV block has occurred after ablation of the fast pathway, patients in this study who underwent fast pathway ablation did not qualify for outpatient management. However, no cases of delayed AV block have been reported after slow pathway ablation, and this complication was not observed in the present study.

In this study, only the right femoral vein was used for the placement of catheters, thereby avoiding the possibility of a pneumothorax associated with subclavian or internal jugular vein cannulation. In patients who undergo cannulation of a subclavian or internal jugular vein, a chest roentgenogram to rule out a pneumothorax would be advisable before discharge from the hospital. In regard to cardiac perforation and the delayed onset of cardiac tamponade, this complication did not occur in the patients in this study or in other reports on outpatient electrophysiologic testing or radiofrequency ablation. ${ }^{5}$

In a previous study, Kalbfleisch et $\mathrm{al}^{1}$ showed a substantial cost advantage of radiofrequency ablation of the fast pathway in patients with AV nodal reentrant tachycardia compared with other forms of therapy. The patients in that study had a mean hospital stay of $3.0 \pm 1.5$ days, and the total charges were $\$ 15,893 \pm 3,338$. In the present study, the mean total charges were reduced to $\$ 10,192 \pm 846$ for patients undergoing slow pathway ablation on an outpatient basis, representing a savings of $36 \%$. Therefore, the results of this study show that a substantial cost savings can be achieved when radiofrequency ablation of the slow pathway is performed on an outpatient basis and that safety is not compromised when early discharge is limited to patients who are appropriate candidates for outpatient therapy.

1. Kalbfleisch SJ, Calkins H, Langberg JJ, El-Atassi R, Leon A, Borganelli M, Morady $F$. Comparison of the cost of radiofrequency catheter modification of the atrioventricular node and medical therapy for drug-refractory atrioventricular node reentrant tachycardia. I Am Coll Cardiol 1992;9:1583-1587.

2. Jackman WM, Beckman KJ, McClelland JH, Wang X, Friday KJ, Roman CA, Moulton KP, Twidale N, Hazlitt A, Prior MI, Oren J, Overholt ED, Lazzara R. Treatment of supraventricular tachycardia due to atrioventricular nodal reentry by radiofrequency catheter ablation of slow-pathway conduction. $N$ Engl J Med 1992; 327:313-318.

3. Haissaguerre M, Gaita F, Fischer B, Commenges D, Montserrat P, D'Ivernois $C$, Lemetayer $P$, Warin JF. Elimination of atrioventricular nodal reentrant tachycardia using discrete slow potentials to guide application of radiofrequency energy. Circulation 1992:85:2162-2175.

4. Kalbfleisch SJ, El-Atassi R, Calkins H, Langberg JJ, Morady F. Safety, feasibility and cost of outpatient radiofrequency catheter ablation of accessory atrioventricular connections. J Am Coll Cardiol 1993;21:567-570.

5. Kadish A, Calkins $\mathbf{H}$, de Buitleir M, Morady F. Feasibility and cost savings of outpatient electrophysiologic testing. J Am Coll Cardiol 1990;16:1415-1419.

\section{Abnormal Hemodynamic Response to Valsalva Maneuver in Mitral Stenosis}

Liang-Miin Tsai, MD, and Jyh-Hong Chen, MD, PhD

A cute hemodynamic response to the Valsalva maneuver has long been used as a means of assessing the circulatory changes in various cardiovascular diseases. ${ }^{1-5}$ An abnormal Valsalva response regarding blood pressure and heart rate changes has been reported in patients with mitral stenosis (MS). ${ }^{4-6}$ However, the central blood flow changes were not determined previously, and hence the potential effects of cardiac stroke

From the Section of Cardiology, Department of Internal Medicine, National Cheng Kung University Medical College and Hospital, 138 Shing Li Road, Tainan, Taiwan 70428, Republic of China. Manuscript received April 27. 1993; revised manuscript received and accepted May $27,1993$. output during this maneuver are poorly understood. Recently, Doppler echocardiography proved to be a useful noninvasive method to evaluate left ventricular stroke output. $^{7-9}$ The purpose of the present study was to evaluate the effect of the Valsalva maneuver on aortic blood flow profiles in patients with MS using the Doppler echocardiographic technique, and to better understand the extent and mechanism of abnormal hemodynamic response to this maneuver in MS based on the beat-tobeat estimation.

Ten patients ( 5 men and 5 women; age range 25 to 43 years, mean 37) with rheumatic MS as documented by physical examination and echocardiography were 